\title{
Umbral de sensibilidad de los nervios periféricos de la mano en pacientes con diabetes tipo II
}

\author{
Amparo Ardila de Chaves* \\ Xiomara Flórez Gamboa** \\ Fiorella Sighinolfi Ramírez*** \\ Gladys Eugenia Villamizar Garzón ${ }^{* * * *}$
}

\section{Resumen}

Las neuropatías periféricas en mano de los pacientes con diabetes tipo II son poco estudiadas por lo que esta investigación de tipo descriptivo se buscó establecer su manifestación mediante una prueba de sensibilidad con monofilamentos de Semmes-Weinstein (SWME) realizada a 66 pacientes que asisten a la Clínica de Diabetes del Hospital Militar Central de Bogotá (HOSMIC), en un período de 4 meses.

Se tuvo en cuenta la edad, el género y la dominancia, las que se relacionaron con el umbral sensitivo en los territorios autónomos de los nervios radial, mediano y cubital. Las 132 manos evaluadas el mayor porcentaje presentó disminución del tacto ligero, así: 43\% nervio radial, $36 \%$ nervio mediano y $42 \%$ nervio cubital. La disminución de la sensación protectiva mostró que el nervio radial se comprometió en el 29\%, el nervio mediano en el $21 \%$ y el cubital en el 14\%. Las mujeres tienen el mayor porcentaje de normalidad en el tacto con $42 \%$, mientras que los hombres lo presentaron en la disminución del tacto ligero en un $43 \%$. En cuanto a edad, el mayor compromiso se presentó en usuarios mayores de 70 años en un $26 \%$ y no hubo notoria diferencia entre la sensibilidad de la mano dominante y la no dominante.

Palabras clave: diabetes tipo 2, neuropatía periférica, monofilamentos de Semmes-Weinstein.

Fisioterapeuta. Especialista en Docencia Universitaria. Especialista en Rehabilitación de Miembro Superior y Mano. ampard@hotmail.com

** Fisioterapeuta. Especialista en Gerencia en Servicios de Salud. Especialista en Rehabilitación de Miembro Superior y Mano.

${ }^{* * *}$ Fisioterapeuta . Especialista en Rehabilitación de Miembro Superior y Mano.

${ }^{* * * * *}$ Fisioterapeuta. Especialista en Docencia Universitaria. Especialista en Rehabilitación de Miembro Superior y Mano. Especialista en Administración Hospitalaria. Hospital Militar Central. 


\title{
Sensitivity threshold of peripheral nerves of the hand in patients with type II diabetes
}

\begin{abstract}
Peripheral neuropathies in hand of patients with diabetes are poorly studied so this research is a descriptive cross, find their expression through a sensitivity test to Semmes-Weinstein monofilament (SWME) performed to 66 patients attending to the Diabetes Clinic of the Central Military Hospital in Bogotá (HOSMIC), over a period of 4 months.

The variables that were considered were age, gender and dominance which were related to sensory findings in the autonomous territories of the radial nerve, median and ulnar.

It was established that 132 of the hands evaluated provided the highest percentage decrease in light touch, and $43 \%$ radial nerve, median nerve $36 \%$ and $42 \%$ ulnar nerve. The decrease of the protective sensation showed that the radial nerve was committed at $29 \%$, the median nerve in $21 \%$ and the ulnar in $14 \%$.

It was concluded that women have the highest percentage of normalcy in touch with $42 \%$ while men showed a decrease in light touch by $43 \%$. Regarding age, the greater was present in users over 70 years by $26 \%$ and there was no noticeable difference between the sensitivity of the dominant hand and non-dominant.
\end{abstract}

Key word: Type 2 diabetes, peripheral neuropathy, of Semmes-Weinstein monofilament.

\section{Introducción}

La diabetes es considerada como una de las enfermedades crónicas más comunes en el mundo. La Organización Mundial de la Salud (OMS) calcula que a nivel mundial hay más de 180 millones de personas con diabetes, y es probable que esta cifra aumente a más del doble en el año 2030. Está entre las diez primeras causas de muerte en la mayoría de los países desarrollados y también en los países en vía de desarrollo como Colombia ${ }^{1}$.

El número de personas que padecen diabetes en América se estimó en 35 millones en el año 2000, de las cuales 19 millones (54\%) vivían en América Latina y el Caribe. Las proyecciones indican que en 2025 esta cifra ascenderá a 64 millones, de las

OPS. La salud en las Américas, Publicación No. 569 de 1998. cuales 40 millones (62\%) corresponderán a América Latina y el Caribe ${ }^{2}$.

En Colombia el II estudio nacional de factores de riesgo de enfermedades crónicas (ENFREC II), publicado en 1999, estimó la prevalencia de diabetes mellitus en la población adulta del país en 2,0\% (IC95\%) 1.28-2-71). Esto correspondería aproximadamente a que 441.000 y 920.000 personas entre 18 y 69 años tienen diabetes mellitus.

Por ser la diabetes una patología que tiene alta incidencia y que a mediano plazo deteriora de manera importante la calidad de vida de quien la padece, es necesario hacer investigaciones que aporten más al conocimiento de dicha enferme-

OMS. La diabetes en las Américas. Boletín Epidemiológico. Junio de 2001, 22 (2). 
dad y de esta forma minimizar los problemas que acarrea ésta en la salud pública.

"No es exagerada la premisa de que la diabetes es una de las principales condiciones que contribuyen a la pérdida de salud y mortalidad prematura a nivel mundial. Es una epidemia silenciosa que tiene el potencial de desestabilizar los servicios de salud de cualquier lugar del mundo"3.

La diabetes mellitus es un síndrome que compromete varios sistemas del organismo cuya característica relevante es el aumento en los niveles de glucosa en sangre dado por fallas en la secreción de insulina, en su acción o en ambos que lleva a trastorno metabólico de carbohidratos, grasas y proteínas.

La insulina es una hormona que regula la presencia de azúcar en la sangre y en la diabetes, este mecanismo regulador se puede comprometer por: deficiencia en su producción o secreción de la misma por el páncreas, o porque el organismo no la puede utilizar eficazmente, lo cual produce elevación excesiva en los niveles de glucemia (hiperglucemia).

La persona con leve hiperglucemia puede ser sintomática por mucho tiempo por lo que se considera necesario hacer actividades de detección precoz sobre todo en población en alto riesgo de padecer diabetes. La persona con marcada hiperglucemia puede presentar síntomas tales como poliuria, polidipsia, polifagia, pérdida de peso, visión borrosa, retardo en el crecimiento (niños) y susceptibilidad para ciertas infecciones. En aquellos individuos con hiperglucemia de mucho tiempo pero sin diagnóstico pueden presentarse con signos y síntomas de complicaciones crónicas de la enfermedad como dolor, ardor o entumecimiento de la parte distal de las extremidades conocida

Unwin, Nigel. Reducing the impact of diabetes on the World's poor. The role of World Health Organization in Geneva and how partners may support its work. Coordination of the joint WHO-IDF programme: Diabetes Action now. 2003. ésta última como neuropatía diabética además de la proteinuria y disfunción eréctil entre otras.

La diabetes se clasifica en dos tipos principales: Tipo I que aparece con mayor frecuencia durante la infancia o la adolescencia y se caracteriza por ausencia de la producción de insulina causada por destrucción de las células Beta que en la mayoría de los casos tiene un factor autoinmune y la Tipo II que puede ser causada, por resistencia periférica a la acción de la insulina que exige una mayor disponibilidad de ésta o, por un defecto en la producción de la insulina que hace que las células Beta no puedan atender la mayor demanda. Está relacionada con la obesidad y la inactividad física, la cual representa el $90 \%$ de todos los casos de diabetes y la cual aparece con mayor frecuencia después de los 40 años de edad, se presenta más en mujeres que en hombres, su aparición es silenciosa y habitualmente no requiere de tratamiento insulínico.

La neuropatía periférica es una de las complicaciones más frecuentes del usuario con diabetes. El nervio se ve afectado en su estructura y en su función y su compromiso inicia con una alteración en la conducción de sensaciones. El individuo pierde la sensibilidad y por tanto, su capacidad protectiva ante estímulos nocivos, lo cual lleva a una serie de cambios morfológicos localizados en las estructuras ligamentarias, articulares y musculares que cambian su biomecánica, aumentando su predisposición a una lesión tisular. Ante ésta el organismo responde con un proceso de reparación y cicatrización, que en el individuo diabético es anormal por la alteración vascular que se da tanto en los grandes como pequeños vasos sanguíneos arteriales haciendo que el tejido no reciba la suficiente sangre oxigenada para realizar su proceso de nutrición, lo cual lleva a cambios irreversibles en el nervio periférico ${ }^{4}$.

\footnotetext{
Wayletn-Rendall, Janeth. Sensibility evaluation and rehabilitation. Orthopedics Clinics of North America. January 1988, 19 (1).
} 
Existen tres tipos de nervios periféricos: los motores, los sensitivos y los mixtos que tienen fibras motoras y sensitivas y además fibras reguladas por el sistema nervioso autónomo. Las fibras nerviosas motoras transmiten señales a los músculos para permitir movimientos como por ejemplo, caminar o hacer movimientos precisos con los dedos. Los nervios sensitivos llevan mensajes en la dirección opuesta y transmiten información acerca de formas, movimientos, texturas, calor, frío o dolor desde los receptores cutáneos especializados que se encuentran en la piel y en el interior del cuerpo, hacia la corteza cerebral.

El sistema sensorial para la percepción está organizado por una serie de cadenas de neuronas que ascienden de la mano al cerebro. Los receptores neuronales responden sólo al estímulo en un área definida de la piel, llamada campo receptor y cada neurona a lo largo de la vía sensorial está también asociada con éste. Si un estímulo es movido, un diferente campo receptor y una serie de neuronas se estimulan para permitir la identificación de un punto de otro porque unas neuronas se activan antes que otras.

Al número de fibras nerviosas que inervan un campo receptor se les conoce como densidad de inervación y ésta determina la cantidad de representación cortical del área en la corteza cerebral. A la vez receptores sensoriales inervan la superficie palmar de los dedos y del antebrazo, además una gran área de la corteza cerebral se representa en la superficie palmar de los dedos y del antebrazo, por lo que los pulpejos de los dedos tienen representación específica de inervación de los nervios periféricos de la mano. La zona autónoma del nervio mediano está ubicada en el pulpejo del dedo índice, la del nervio cubital en el pulpejo del meñique y la del nervio radial en la cara dorsal del primer espacio interóseo.

Las lesiones de nervio periférico pueden alterar la percepción sensorial de la mano, comprometiendo su función, por lo que es necesaria la habilidad en la evaluación de la sensibilidad por ser de gran ayuda para el terapeuta y el médico en el tratamiento de pacientes con neuropatías periféricas.

La información obtenida acerca de la sensibilidad de cada paciente ayuda a clarificar la incapacidad funcional e identificar la terapia necesaria, porque cuando ésta se compromete se disminuye y limita su función, se disminuye la ejecución de los movimientos y la manipulación precisa y veloz de los objetos.

En un test de sensibilidad se puede despolarizar la membrana mecánicamente y el voltaje de la membrana se incrementa con el aumento del flujo de iones hasta que es lo suficientemente fuerte para alcanzar el umbral del axón y disparar el potencial de acción, autopropagado a lo largo del nervio. Todos los receptores sensitivos cutáneos y sus fibras asociadas se adaptan a un estímulo aplicado y pueden hacerlo despacio o rápidamente. Los receptores sensoriales transducen los estímulos hasta el impulso eléctrico llamado potencial de acción. La despolarización resulta de un incremento continuo de iones de sodio a través de la entrada en el receptor de la membrana celular que puede despolarizarse por un impulso eléctrico, deformación mecánica o un mensaje químico.

Cuando hay una lesión nerviosa hay varios factores que pueden influenciar el diámetro de la fibra o su mielinización y afectar la velocidad de conducción. El daño de la vaina de mielina causa disminución de la velocidad de conducción y disminuye progresivamente hasta la desmielinización completa. Después de la reparación nerviosa, las fibras regeneradas conducen impulsos más lentamente porque son delgadas y no recuperan su diámetro original o su completa mielinización por muchos años.

El diagnóstico de la neuropatía diabética es factible por medio de pruebas evaluativas específicas de sensibilidad, las cuales se dividen en tres cate- 
gorías: pruebas de umbral, pruebas funcionales y pruebas simpáticas ${ }^{5}$.

Los test de sensibilidad son un importante componente de todas las evaluaciones de la mano; porque la sensibilidad es esencial en su función. Dentro de éstos los más pertinentes para la evaluación de la sensibilidad en los diabéticos están las pruebas de umbral, las cuales evalúan la sensibilidad superficial que incluye la percepción de temperatura, la presión ligera y profunda además de la vibración 6 .

Una de las formas de evaluar la presión ligera y profunda es por medio de los monofilamentos de Semmes-Weinstein, por medio de los cuales se puede establecer el umbral de sensibilidad de cada nervio periférico.

Esta prueba tuvo su origen en 1898 cuando el fisiólogo alemán Max Von Frey, desarrolló una prueba de umbrales de tacto y enfocó su estudio de la fisiología normal donde sólo se medía el tacto fino; descubrió que la presión de una crin de caballo contra una superficie ocasiona una incurvación con la particularidad de que la curvatura que se produce no es proporcional a la presión ejercida, propiedad que consideró muy útil para evaluar de la sensación táctil ${ }^{7}$.

En 1950, Josephine Semmes y Sydney Weinstein, basándose en el estudio mencionado desarrollaron un amplio rango de 20 monofilamentos de diámetros diferentes y de longitud constante que remplazan los de Von Frey para medir los umbrales de fuerza de presión relativa.

Tubiana, Raoul. Examination of the hand and wrist. Mosby. 1a. ed., 1998, p. 328.

6 Meijer, Jan Willem y cols. Clinical diagnosis of diabetic polyneuropathy with the diabetic neuropathy symptom and diabetic neuropathy examination scores. Diabetes Care. Vol. 26, Number 3. March 2003.

Monofilaments for evaluating sensory neuropathy. July 28 , 2004. En: http://www.cbi pace.com/ monofilaments.com
En 1960, Semmes y Weinstein describieron las fuerzas características de esta batería de monofilamentos que insertaron en mangos de plástico, y que denominaron posteriormente como "Aestesiómetros de Semmes-Weinstein (ASW)".

En 1967 Von Prince introduce y acredita el uso de estos monofilamentos en la clínica y cuantifica mediante progresión logarítmica los niveles de presión de cada uno; esta fuerza aplicada por cada uno de los monofilamentos, se expresa mediante tres dígitos, que indican el logaritmo de la presión ejercida en gramos, más una constante equivalente a 4 .

Con base en esta batería, en 1978, Bell desarrolló una de 5 monofilamentos, considerados como los más representativos para la predicción de cambios sensoriales.

En 1995, Weinstein mejoró y patentó el aestesiómetro que ha sido adoptado como estándar. Esta prueba evaluativa (Weinstein Enchanced Sensory Test, o WEST), ha sido probada en varios estudios para evaluar la pérdida de sensación táctil en personas diabéticas y así detectar el riesgo de ulceración que presentan las complicaciones neuropáticas ${ }^{8}$.

Los monofilamentos de Semmes-Weistein, son fibras de nylon calibradas de tal manera que al aplicarlos en la piel hay una presión determinada, la cual es independiente de la curvatura generada por la presión. De esta manera, pequeñas vibraciones o movimientos de la mano del examinador no influyen sobre la cantidad de fuerza ejercida ${ }^{9}$. Se identifican con números asignados por sus fabricantes en un rango de 1.65 a 6.65 , que se derivan la siguiente fórmula: Valor nominal $=\log 10$ (fuerza $(\mathrm{mg}) * 10)$.

Ibíd.

Mayfield, J.A. The use of the Semmes- Weinstein and other threshold tests for preventing foot ulceration and amputation in persons with diabetes. J Fam Pract. 2000, 49 (suppl): pp. 17-29. 
La longitud de los monofilamentos es constante $(38 \mathrm{~mm})$, mientras que el diámetro varía entre 0.635 y $1.143 \mathrm{~mm}^{10}$. En la batería de cinco monofilamentos el de color verde $(1.65-2.83)$ indica que el tacto ligero es normal, el de color azul (3.22 - 3.61) informa la disminución del tacto ligero, el color violeta (3.84 - 4-31) indica disminución de la sensibilidad protectiva. El de color rojo claro $(4.56$ - 6.65) muestra pérdida de la sensación protectiva y el rojo oscuro (>6.65), permite establecer la pérdida de la sensibilidad o anestesia.

Esta prueba sigue parámetros específicos para su aplicación porque requiere de la colaboración del paciente, a quien se le explica el método y se ensaya sobre su antebrazo para posteriormente, con ojos vendados reconocer el estímulo del monofilamento aplicado de forma perpendicular a la piel evaluada, por 1 segundo ${ }^{11}$. De acuerdo con estudios sensoriales comparativos sobre diferentes superficies, este examen puede seguir dos métodos: uno en el cual se aplica la fuerza en momentos diferentes sobre un mismo sitio previamente establecido; el paciente debe responder si siente el monofilamento (sí/no) y el examinador registra las respuestas que sean correctas y calcula el número de errores; ya que umbral de sensación protectiva se define en este caso mediante el total de momentos de aplicación del monofilamento no percibidos por la persona ${ }^{12}$.

Es importante resaltar que como para este examen el tiempo de evaluación es mínimo, se facilita su aplicación a pacientes en consulta externa, lo cual permite control permanente de la evolución del nervio comprometido.

10 Sumpio, B.E. Foot Ulcers. The new England Journal of Medicine. 2000, 343: pp. 783-793

11 Lee, Sangyeoup y cols. Clinical Usefulness of Two-site Semmes-Weinstein Monofilament Test for Detecting Diabetic Peripheral Neuropathy. Journal Korean Medical Science. Korea. Vol. 18, 2003, pp. 103-107.

12 Dimitrakoudis, Bril V. Comparation of sensory testing on different toe surfaces: Implications for neuropathy screening. Neurology, 2002, 59: pp. 611-613.
Es muy amplia la bibliografía sobre evaluación de sensibilidad del pie de la persona con diabetes mediante las diferentes pruebas referidas pero es muy escasa la que hace referencia de sensibilidad en miembro superior como complicación de dicha patología. Valk en $1999^{13}$, hizo la validación de la prueba de monofilamentos para evaluar el compromiso de función en alteraciones sensoriales.

Diferentes investigadores han utilizado estos monofilamentos como uno de los instrumentos más confiables para establecer el umbral de sensibilidad en deterioros sensitivos por diferentes patologías. Janeth Waylet-Rendall y cols. ${ }^{14}$ los utilizaron con el método de "sí/no" cuando se aplicaba cada filamento, y Perkins BA y cols. ${ }^{15}$, aplicaron este mismo test en conjunto con el de vibración mediante el método on/off, con los mismos objetivos evaluativos. Un estudio realizado por Brown y cols. $(1984)^{16}$ determinó alteraciones sensitivas y motoras de diferentes nervios mixtos en usuarios con diabetes tipo 2 mediante test de función, pero no fue posible conocer las del nervio ulnar.

Nozomu Kamei y cols. ${ }^{17}$ establecieron la efectividad del examen sensitivo periférico de fibras pequeñas del usuario diabético, con monofilamentos de Semmes Weinstein. Luego de aplicar las pruebas y darse una respuesta sensitiva anormal en cuanto a debilidad, determinó que había alteración de las fibras sensitivas pequeñas.

13

14 Ibídem.

15 Perkins, B.A. Simple screening tests for peripheral neuropathy in the diabetes clinic. Diabetes Care. Feb: 2001, 24 (2): 250256.

16 Brown, M.J. and Asbury, A.K. Diabetic neuropathy. Annals Neurology. 1984, pp. 2-12.

17 Kamei, Nozomu y cols. Effectiveness of Semmes-Weinstein monofilament examination for diabetic peripheral neuropathy screening. Journal of Diabetes and its Complications. JanuaryFebruary 2005, pp. 47-53. 
Se encuentran muchos estudios en los que se fundamenta y valida la prueba de monofilamentos para establecer alteraciones de sensibilidad distal en miembros inferiores. Kamei y cols. $(2005)^{18}$, evaluaron la sensibilidad y especificidad de dos monofilamentos diferentes y sugirieron que la combinación de los sitios 1 (el hallux) y 3 (aspecto plantar de la cabeza del V metatarsiano) fue la más sensible. Correlacionaron los resultados obtenidos con los síntomas subjetivos, con la sensación vibratoria y los reflejos profundos. La prueba de oro fue este examen clínico.

Estos autores utilizaron los SWME (monofilamentos de 4.31/2 y 5.07/10 gramos) y las pruebas de vibración (prueba cuantitativa del diapasón C-64), para evaluar los pies diabéticos y al final se pudo diagnosticar neuropatía diabética periférica cuando 2 de los 3 criterios se encontraron en ambos miembros inferiores: 1. presencia de dolor, entumecimiento, o parestesia. 2. disminución o ausencia del reflejo tendinoso o 3. escala por debajo de 4 para los umbrales de percepción de la vibración (VPT).

Lo anterior muestra la efectividad de la prueba en miembros inferiores pero en miembros superiores se hace necesaria la ejecución de estudios para determinar si hay o no esta neuropatía en el diabético con el fin de prevenir el compromiso del tejido tegumentario de la mano que puede llevar a amputaciones.

Por esta falencia se quiere conocer el umbral de sensibilidad de los nervios periféricos de la mano de los usuarios con diabetes tipo 2 en una población diagnosticada con anterioridad al estudio, con el fin de prevenir complicaciones sensitivas a largo plazo o si ya se presentan disminuir los riesgos de compromisos mayores.

\footnotetext{
${ }^{18}$ Op. cit. Kamei, pp. 47-53.
}

El objetivo del presente estudio fué identificar el umbral de sensibilidad que tienen los nervios periféricos de la mano de los usuarios con diabetes tipo 2 que asisten a la Clínica de Diabetes en el Hospital Militar Central.

\section{Metodología}

\section{Tipo de estudio: Descriptivo}

Población: 66 usuarios con diabetes tipo 2 que asistían a la Clínica de Diabetes del Hospital Militar Central de Bogotá, en agosto, septiembre y octubre de 2007.

Se excluyeron los usuarios con lesiones que comprometían los nervios periféricos que inervan la mano, tales como patología del túnel del carpo o del canal de Guyón. Igualmente los que presentaron amputación de los dedos índice y/o meñique de una o ambas manos.

\section{Instrumentos}

- Batería de cinco monofilamentos de SemmesWeinstein.

\section{Procedimiento}

- Inicialmente se informó a los pacientes respecto de la investigación, en cuanto a su participación y procedimiento a realizar.

- Se resolvieron los interrogantes que surgieron de la información suministrada y firma del consentimiento informado.

- Se aplicó posteriormente a cada paciente del formato diseñado para la recolección de los datos.

- Se aplicó al paciente de los monofilamentos, con ojos abiertos y luego con ojos vendados, primero en la mano dominante y luego en la contraria, en el territorio autónomo de cada nervio periférico. 
- Finalmente se realizó la aplicación secuencial de cada monofilamento, por color, hasta su percepción por parte del paciente y posterior registro de resultados en elformato de evaluación.

\section{Resultados}

Se evaluaron 39 mujeres y 29 hombres. Dos usuarios fueron excluidos por no cumplir con los criterios de inclusión, por lo cual el total de los usuarios que ingresaron al estudio fue de 38 mujeres equivalente al $57 \%$ y 28 hombres con el $43 \%$ para un total de 66.

La mayor frecuencia de edad de los usuarios evaluados con diabetes tipo 2 estuvo en el rango entre 70 y 74 años con $12 \%$ (8 mujeres) y $14 \%$ ( 9 hombres), seguido por el rango de más de 75 años con $8 \%$ (5 mujeres) y $11 \%$ ( 7 hombres).

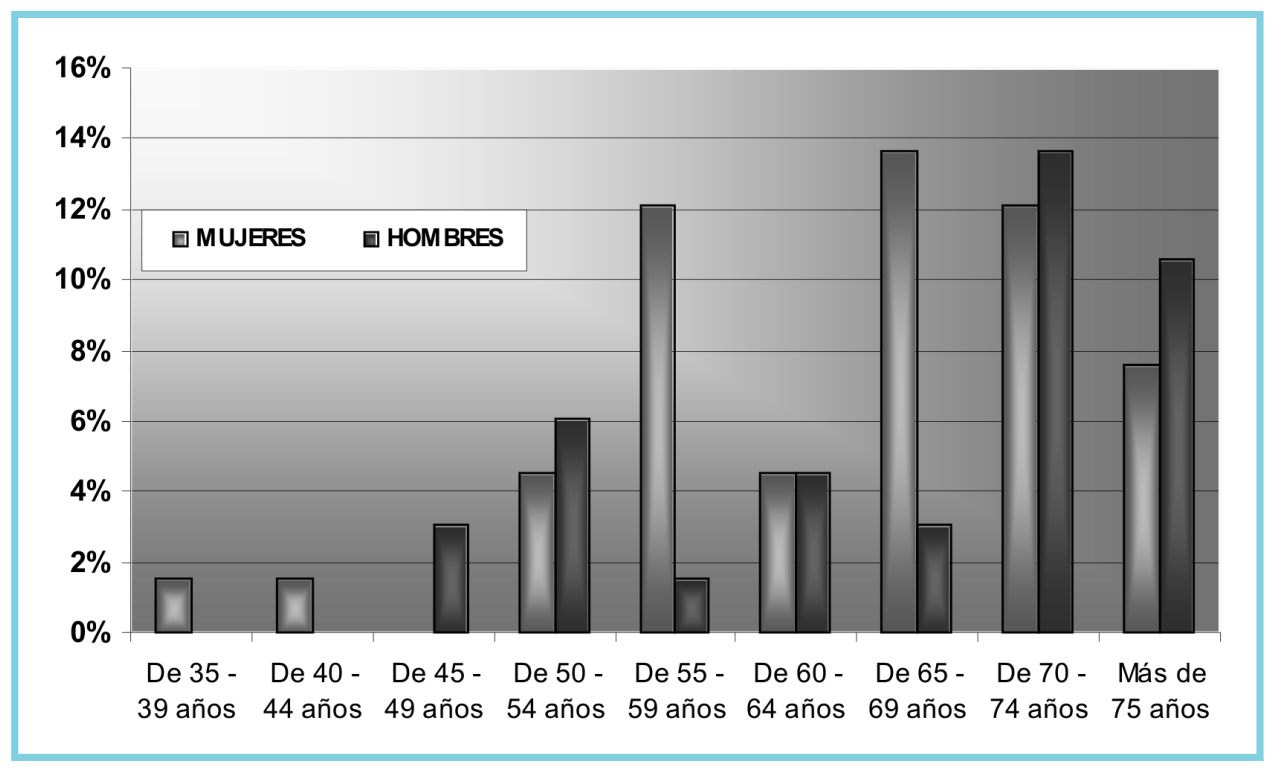

Gráfica 1. Usuarios con diabetes tipo 2 evaluados en HOSMIC - 2007, según género y edad

Luego de la evaluación de umbral de sensibilidad de los usuarios con diabetes tipo 2 en el HOSMIC, se encontró que el nervio cubital tiene el rango más alto en lo referente al tacto normal con $44 \%$ (58 manos), seguido del nervio mediano con un $39 \%$ (52 manos).
Se observó que el mayor compromiso en la disminución del tacto ligero tiene rangos similares pues el mayor porcentaje lo presenta el nervio radial con un $43 \%$ (57 manos), el cubital con un $42 \%$ (55 manos) y el mediano con $36 \%$ (48 manos). 


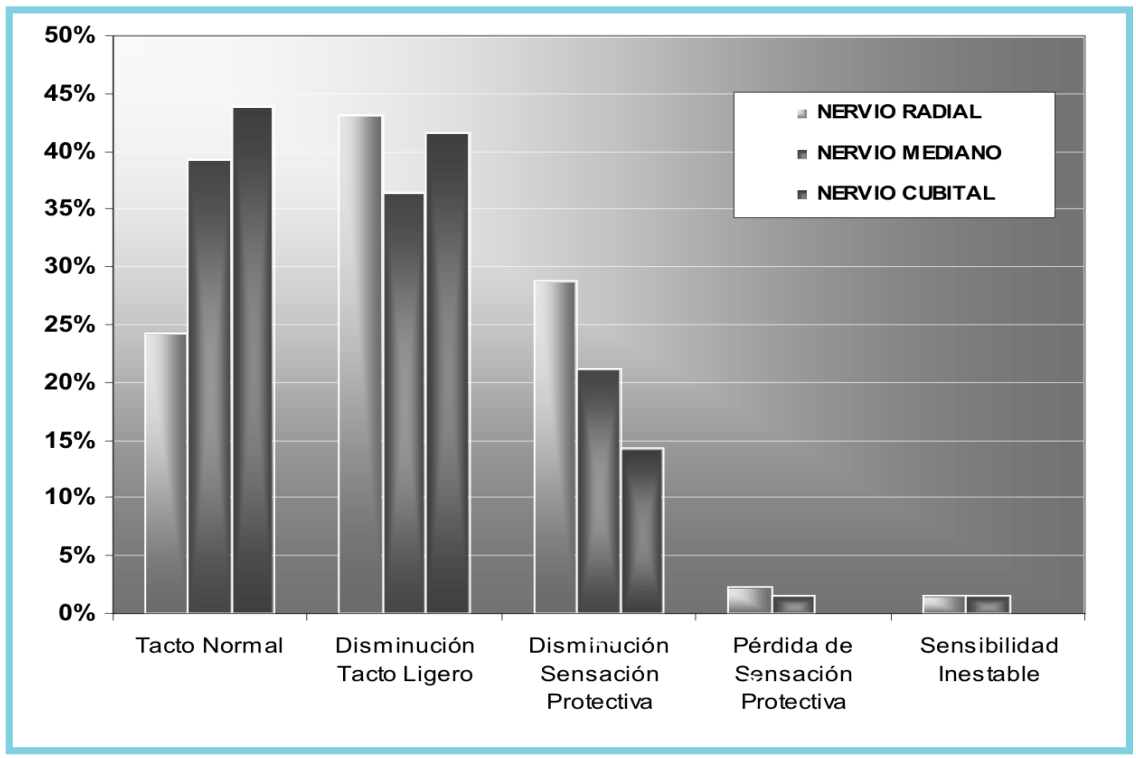

Gráfica 2. Umbral de sensibilidad de los nervios periféricos de la mano en usuarios con diabetes TIPO 2 en HOSMIC - 2007.

La disminución de la sensación protectiva mostró que el nervio radial es el de mayor compromiso con 29\% (38 manos), seguido por el nervio mediano con $21 \%$ (28 manos) y por el cubital con $14 \%$ (19 manos).

La sensación protectiva y la sensibilidad inestable no mostraron compromiso relevante.
En cuanto al umbral de sensibilidad del nervio cubital derecho se encontró que el $29 \%$ de las mujeres (19) y el $11 \%$ de los hombres (7) tienen tacto normal. El 23\% de las mujeres (15) y el $21 \%$ de los hombres (14) tienen disminución del tacto ligero. Presentaron disminución de la sensación protectiva el $6 \%$ de las mujeres (4) y el $11 \%$ de los hombres (7).

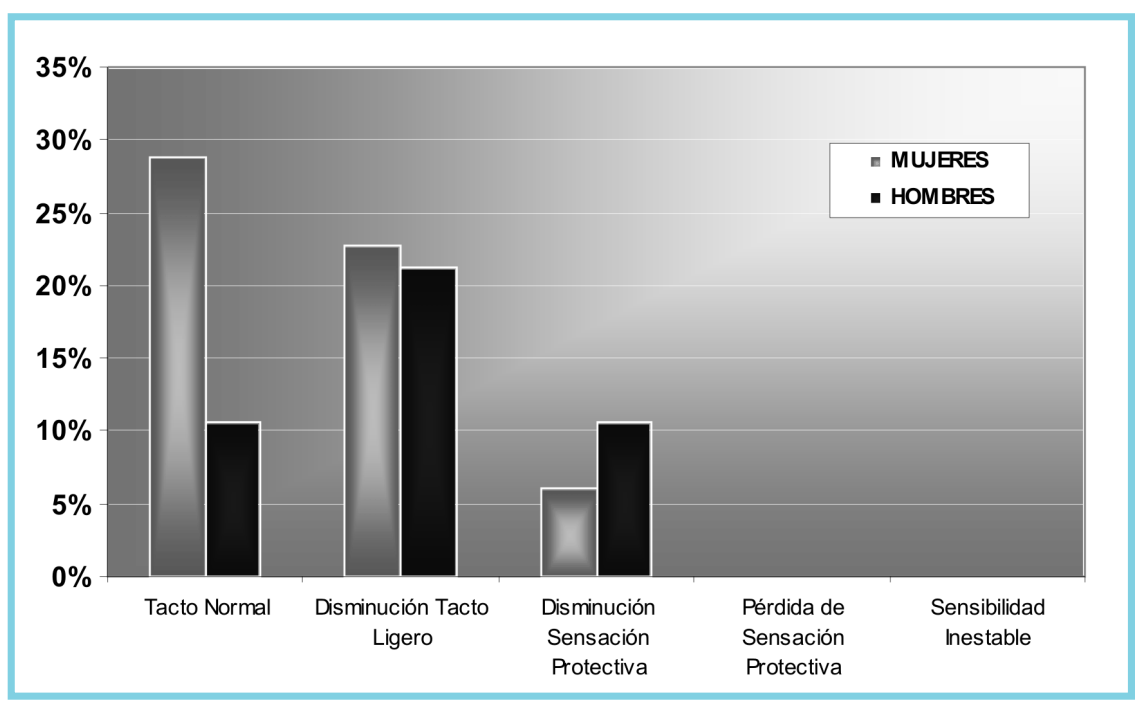

Gráfica 3. Umbral de sensibilidad del nervio cubital derecho y su relación con género. 
Al evaluar el umbral de sensibilidad del nervio cubital izquierdo se encontró que el 32\% de las mujeres evaluadas (21) y el $17 \%$ de los hombres (11) tienen tacto normal, seguido de la disminución del tacto ligero con igual porcentaje de $20 \%$ para hombres y mujeres (13). El 6\% de los hombres y las mujeres (4) presentaron disminución de la sensación protectiva.
Los resultados analizados sobre el umbral de sensibilidad del nervio mediano derecho mostraron que el $26 \%$ de las mujeres (17) y el $14 \%$ de los hombres (9) tienen tacto normal. El 17\% de las mujeres (11) y el 14\% de los hombres (9) presentan disminución del tacto ligero. El 12 de las mujeres (8) y el $14 \%$ de los hombres (9) presentaron disminución de la sensación protectiva y un $2 \%$ de hombres (1) y mujeres (1) mostraron pérdida de la sensación protectiva.

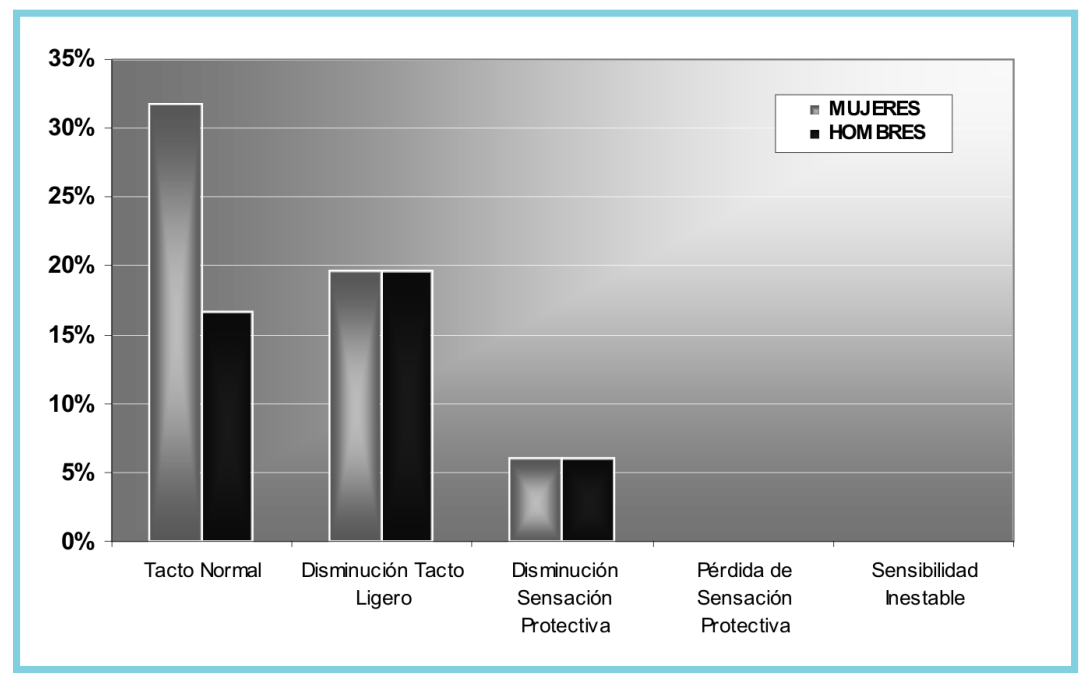

Gráfica 4. Umbral de sensibilidad del nervio cubital izquierdo y su relación con género.

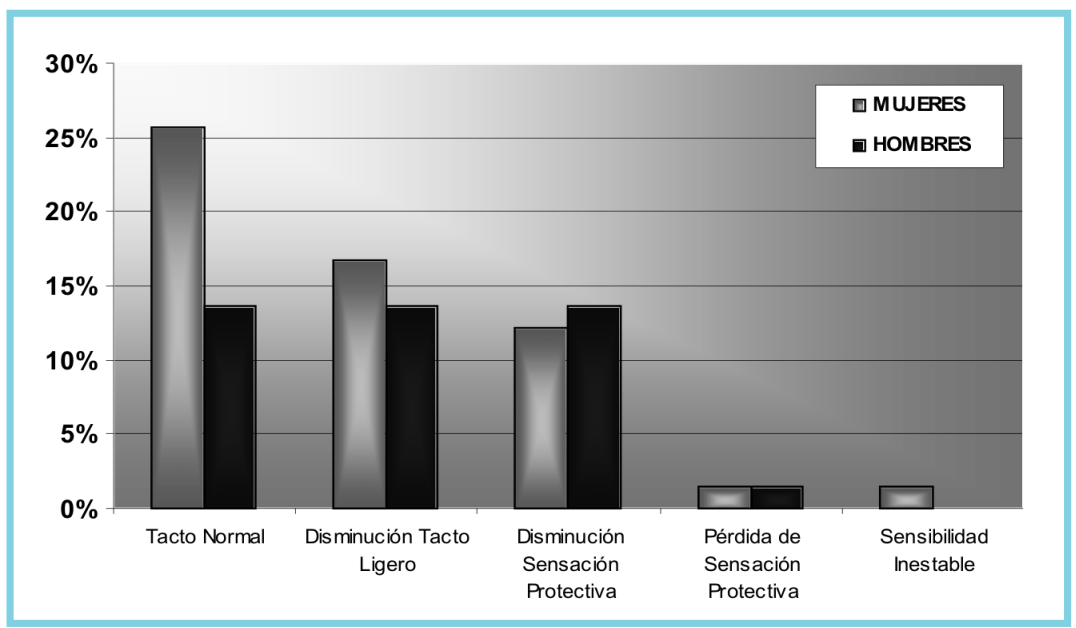

Gráfica 5. Umbral de sensibilidad del nervio mediano derecho y su relación con género. 


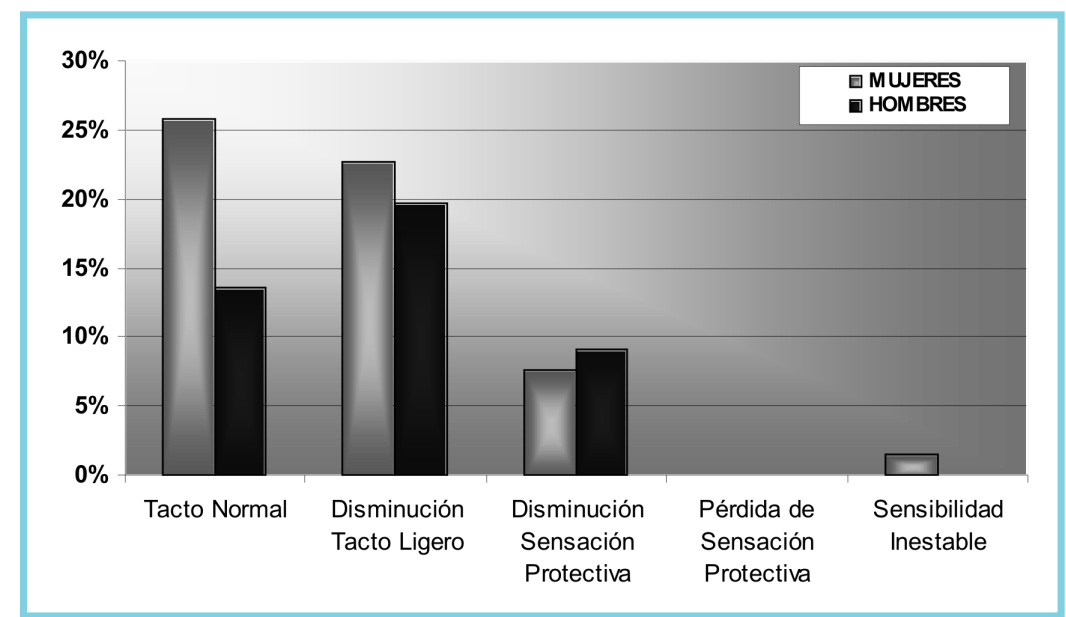

Gráfica 6. Umbral de sensibilidad del nervio mediano izquierdo y su relación con género.

El umbral de sensibilidad del nervio mediano izquierdo dio como resultado que el $26 \%$ de las mujeres (17) y el $14 \%$ de los hombres (9) tienen tacto normal y disminución del tacto ligero el $23 \%$ de las mujeres (15) y el 20\% de los hombres (13). Se encontró que el $8 \%$ de las mujeres (5) y el $9 \%$ de los hombres (6) tuvieron disminución de la sensación protectiva. El $2 \%$ presentó sensibilidad inestable.
En la evaluación del umbral de sensibilidad del nervio radial derecho se encontró que la disminución del tacto ligero fue de $24,2 \%$ en mujeres y $16,7 \%$ en hombres, seguido por la disminución de la sensación protectiva con $18,2 \%$ y $12,1 \%$, respectivamente. Presentaron tacto normal el 18,2\% de las mujeres y el $6,1 \%$ de los hombres. El 1,5\% mostró pérdida de la sensación protectiva y una mujer $(1,5 \%)$ tuvo sensibilidad inestable.

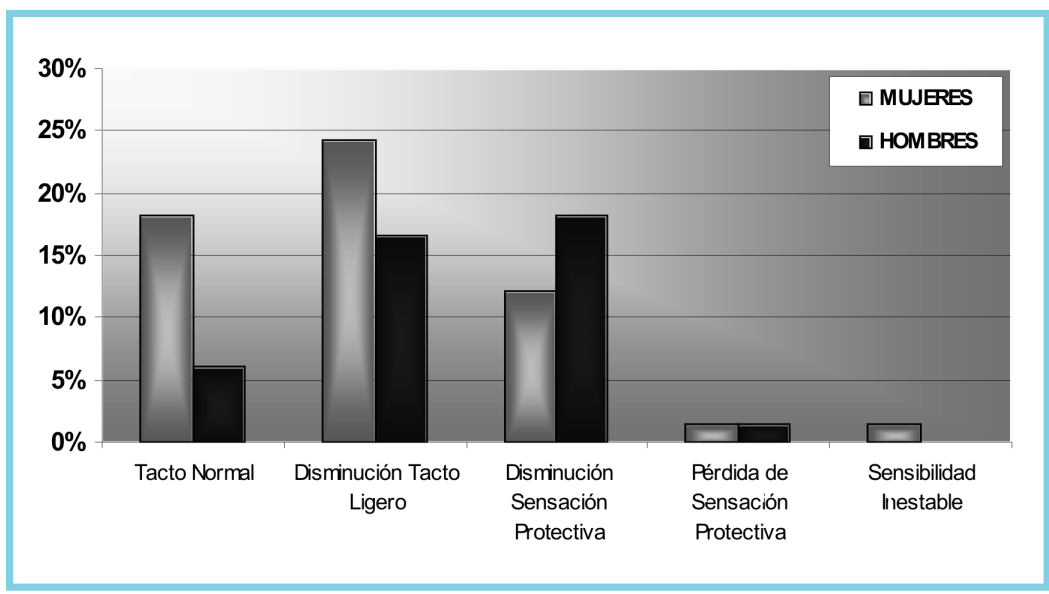

Gráfica 7. Umbral de sensibilidad del nervio radial derecho y su relación con género. 


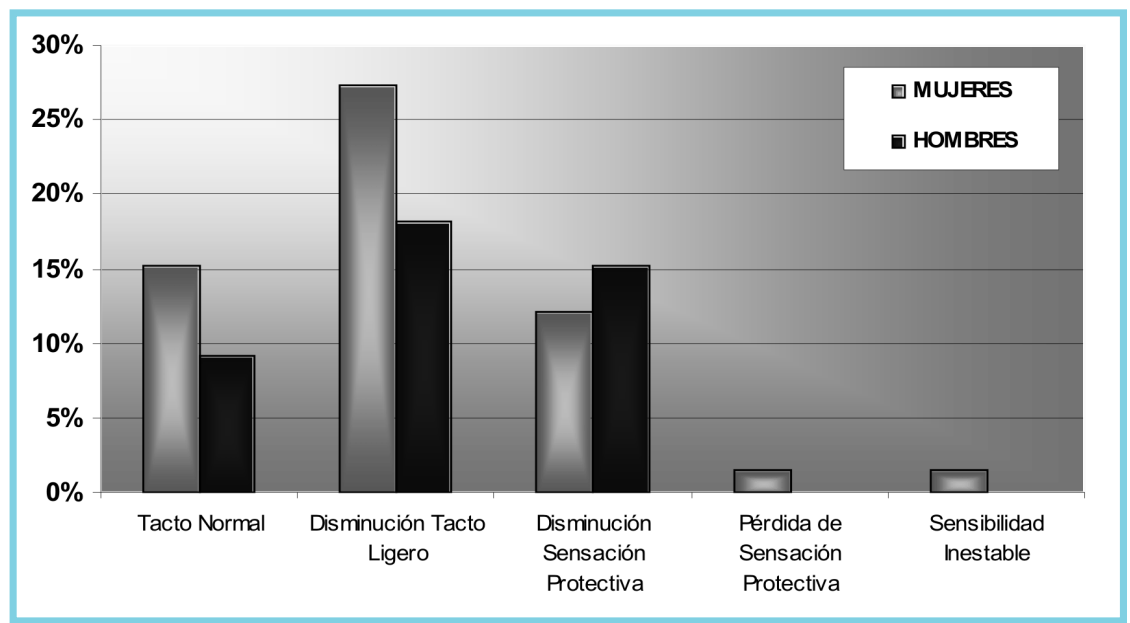

Gráfica 8. Umbral de sensibilidad de nervio radial izquierdo y su relación con género.

En el nervio radial izquierdo se encontró que el $27,3 \%$ de las mujeres (18) y el $18,2 \%$ de los hombres (12) tuvieron disminución del tacto ligero, seguido por la disminución de la sensación protectiva con $12,1 \%$ de mujeres (8) y $15,2 \%$ de los hombres (10). Se encontró que el $15,2 \%$ de las mujeres (10) y un $9,1 \%$ de los hombres (6), presentan tacto normal. Con porcentaje igual de $1,5 \%$ (1) las mujeres presentaron pérdida de la sensación protectiva y sensibilidad inestable.
Respecto de la edad y el umbral de sensibilidad se encontró que del total de todo el proceso evaluativo, el mayor porcentaje de tacto normal está en el rango de edad entre 65 y 69 años, con 9\%. La disminución del tacto ligero tiene su mayor proporción en usuarios entre 70 y 74 años de edad, con $13 \%$. La disminución de sensación protectiva con $8 \%$ fue el porcentaje más alto en usuarios mayores de 75 años y en usuarios mayores de 70 años se encontró pérdida de la sensación protectiva en el $4 \%$ y con porcentaje similar, sensibilidad inestable en el 3\% de la población.

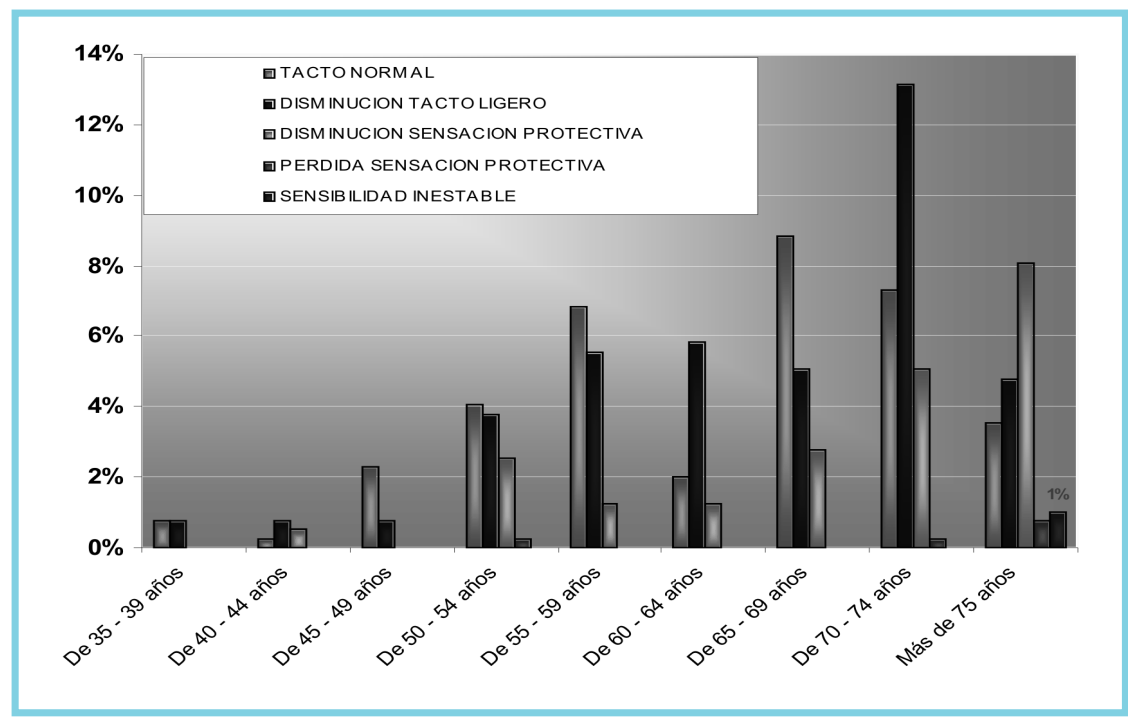

Gráfica 9. Umbral de sensibilidad y su relación con edad. 


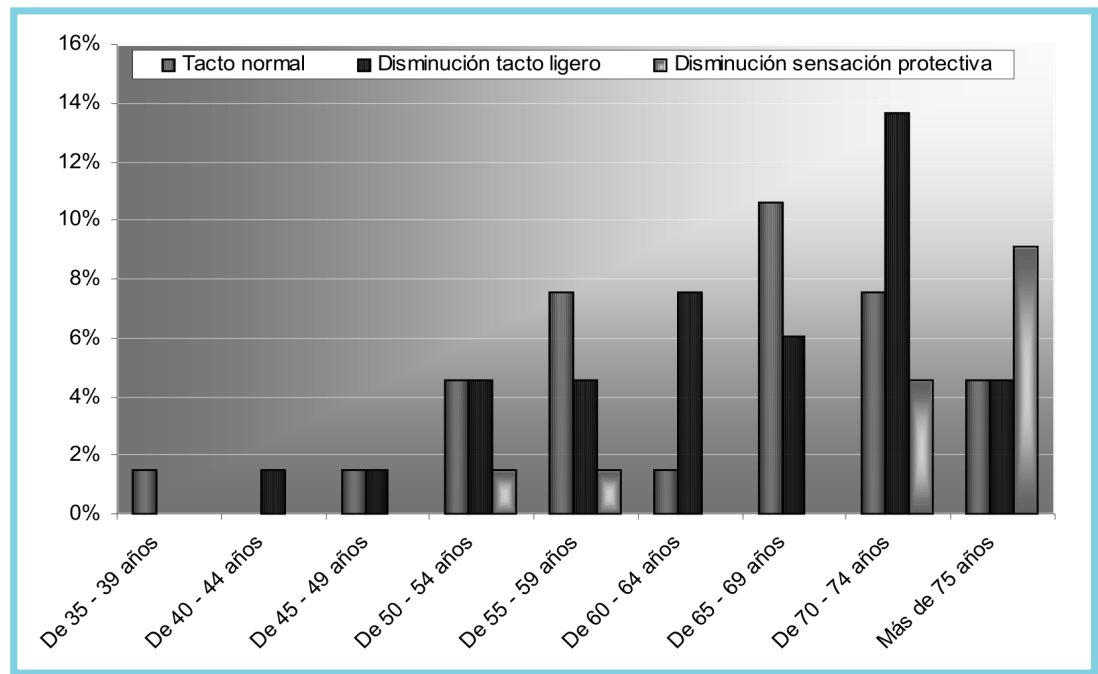

Gráfica 10. Umbral de sensibilidad del nervio cubital derecho y su relación con edad.

En la evaluación del nervio cubital derecho se encontró que el $14 \%$ de la disminución del tacto ligero estuvo en el rango de edad entre 70 y 74 años. El tacto normal tuvo su mayor porcentaje entre los 65 y los 69 años y la disminución de la sensación protectiva con más usuarios comprometidos estuvo en el rango de mayores de 75 años con $9 \%$.
Luego de la evaluación del nervio cubital izquierdo se encontró que en igual porcentaje de $12 \%$ estuvieron el tacto normal y la disminución del tacto ligero en el rango de edad entre 70 y 74 años. El mayor porcentaje del tacto normal se presentó en el rango de edad entre 65 y 69 años con un $12 \%$ y en el rango de edad entre 60 y 64 años se encontró que en un $6 \%$ hay disminución de la sensación protectiva.

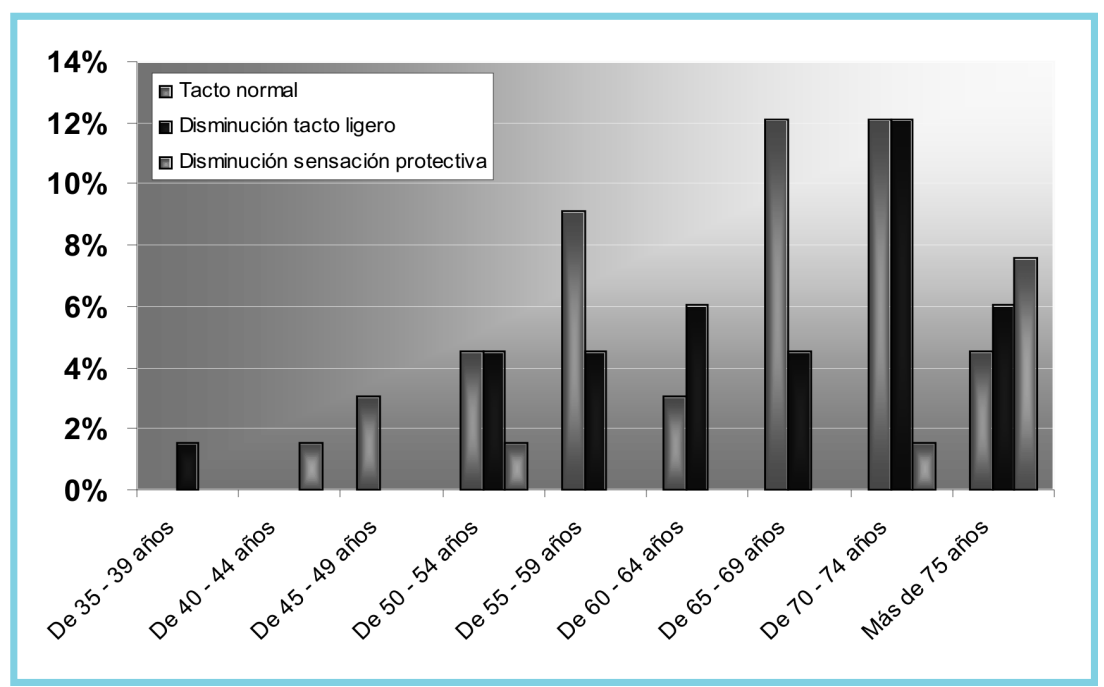

Gráfica 11. Umbral de sensibilidad del nervio cubital izquierdo y su relación con edad. 


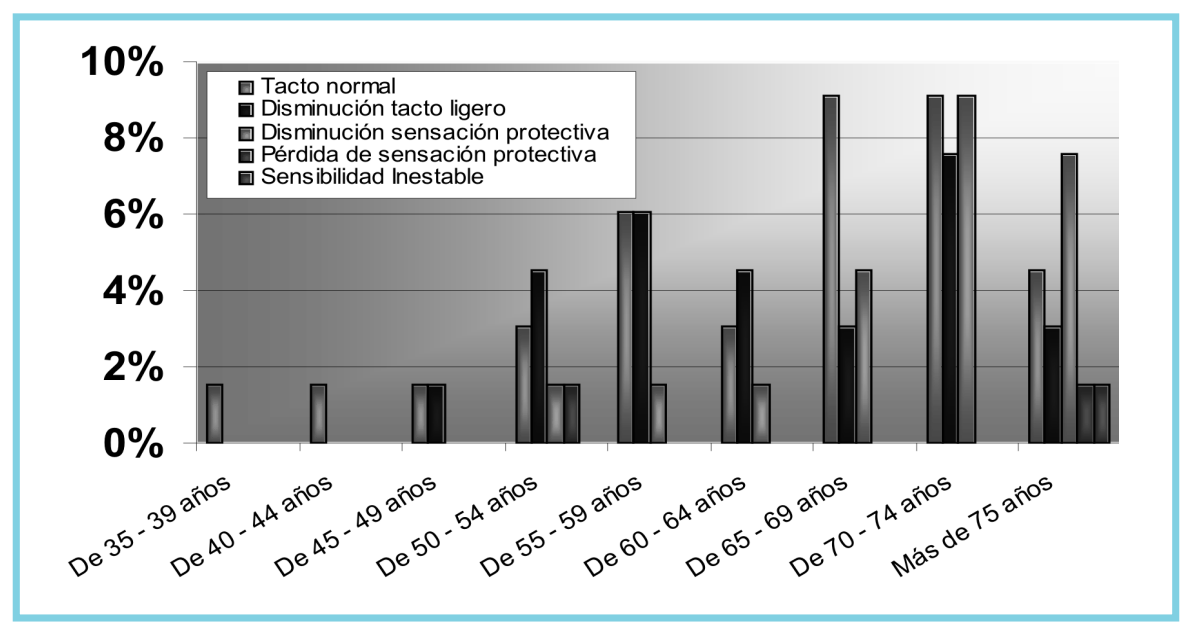

Gráfica 12. Umbral de sensibilidad del nervio mediano derecho y su relación con edad.

Al evaluar el umbral de sensibilidad del nervio mediano derecho se encontró que hay porcentaje igual de $9 \%$ en la disminución de la sensación protectiva y el tacto normal en el rango de edad entre 70 y 74 años y en el mismo rango de edad el $8 \%$ presentó la disminución del tacto ligero. En el rango de edad de 65 a 69 años se encontró que 9\% presentó tacto normal, 5\% disminución de la sensación protectiva y el 3\% disminución del tacto ligero. El rango de usuarios mayores de 75 años mostró que el $8 \%$ tenía disminución de la sensación protectiva, el $5 \%$ tacto normal, $3 \%$ disminución de la sensación protectiva y $2 \%$ igual para pérdida de la sensación protectiva y sensibilidad inestable.

El resultado de la evaluación del nervio mediano izquierdo mostró que el $18 \%$ de los usuarios con disminución del tacto ligero están en el rango de edad entre 70 y 74 años, el más alto porcentaje de tacto normal estuvo en el rango de edad de 65 a 69 años con $12 \%$ y en el rango de edad entre 60 y 64 años el mayor porcentaje lo tuvo la disminución del tacto ligero con $8 \%$. En los mayores de 75 años el porcentaje más alto fue la disminución de la sensación protectiva con $8 \%$.

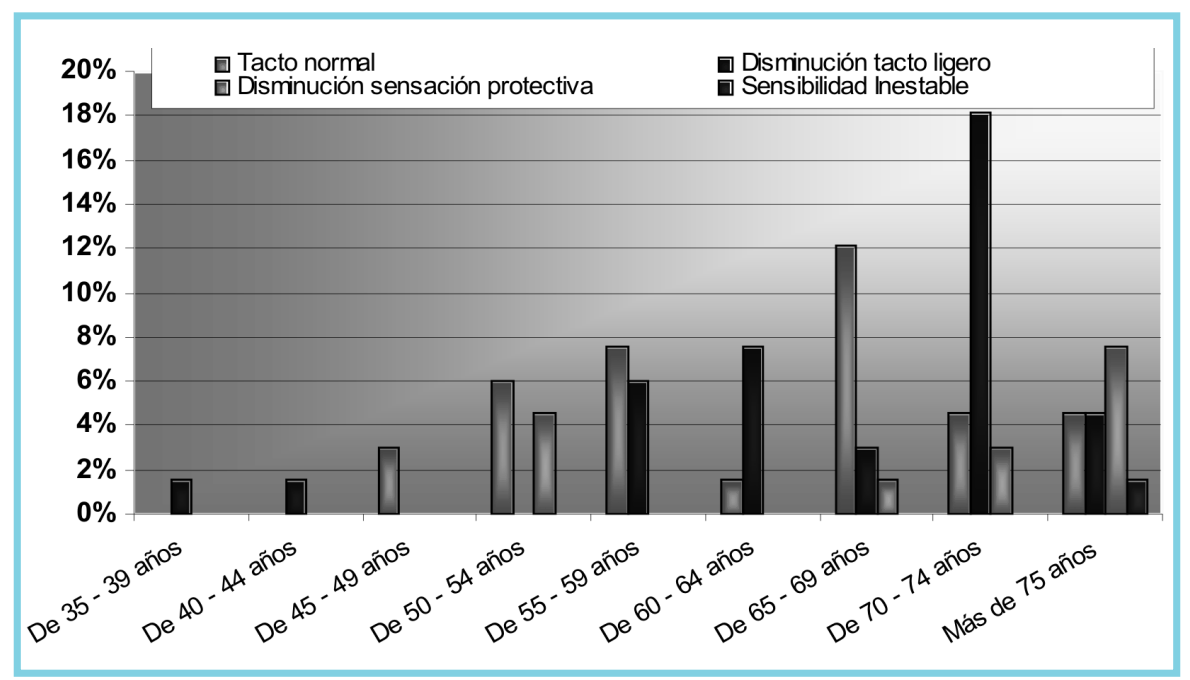

Gráfica 13. Umbral de sensibilidad del nervio mediano izquierdo y su relación con edad. 


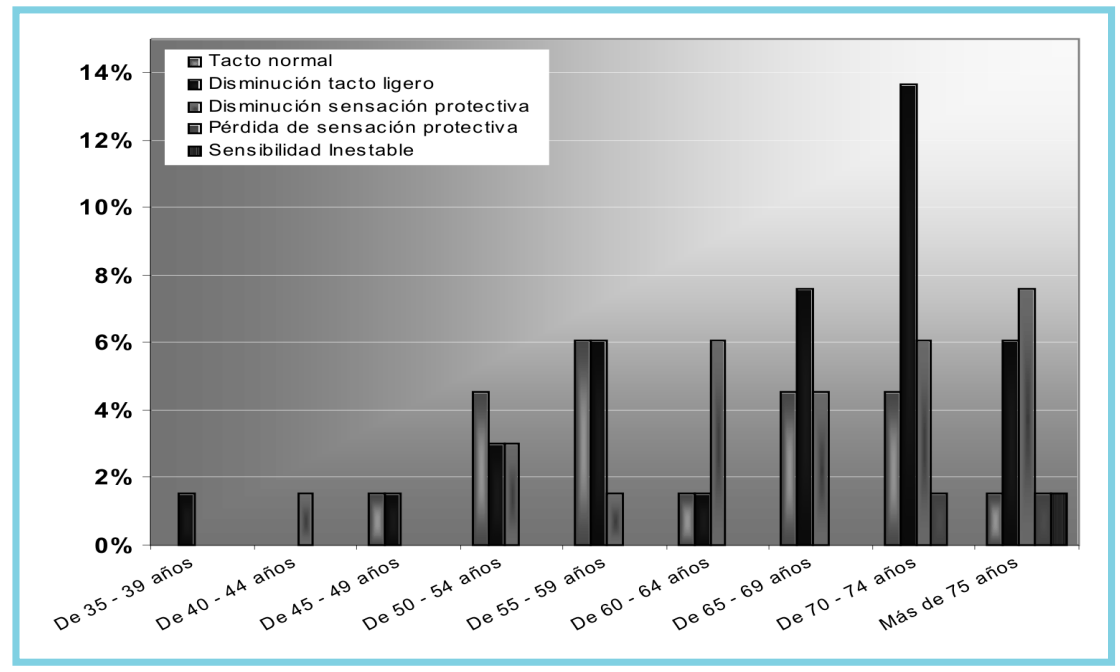

Gráfica 14. Umbral de sensibilidad del nervio radial derecho y su relación con edad.

En la evaluación del nervio radial derecho se encontró que el mayor porcentaje estuvo en la disminución del tacto ligero con 14\% en el rango de edad entre los 70 a 74 años y le sigue la disminución de la sensación protectiva en el rango de mayores de 75 años con un porcentaje de $8 \%$. En el rango de edad entre 65 y 69 años el $8 \%$ presentó disminución del tacto ligero y el $5 \%$ pérdida de la sensación protectiva. Es importante resaltar que en usuarios mayores de 75 años hay mayor compromiso sensitivo y se presentó sensibilidad inestable en usuarios mayores de 70 años.
En la evaluación del nervio radial izquierdo se encontró que el mayor porcentaje se dio en la disminución del tacto ligero en el rango de los 70 a 74 años equivalente al $14 \%$ de la población y le sigue la disminución de la sensación protectiva en el rango de mayores de 75 años con un porcentaje de $9 \%$. En el rango de edad entre 65 y 69 años el $8 \%$ presentó disminución del tacto ligero. Es importante resaltar que en usuarios mayores de 75 años hay mayor compromiso sensitivo específicamente en cuanto a la disminución de la sensación protectiva con $9 \%$. En este mismo rango se presentó pérdida de la sensación protectiva y sensibilidad inestable en $2 \%$.

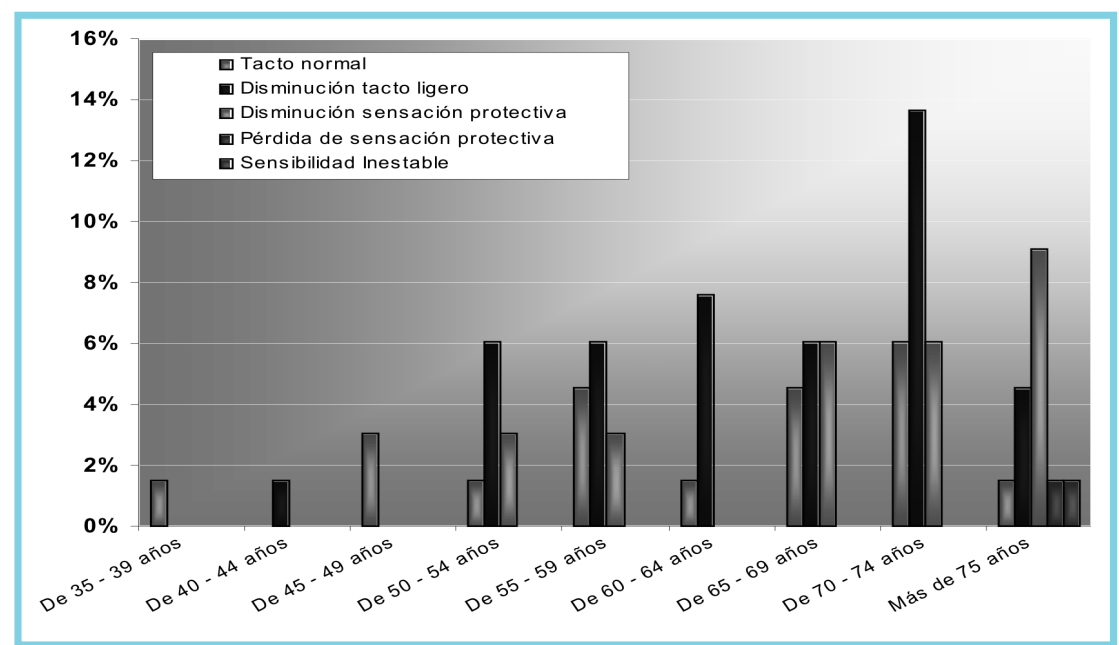

Gráfica 15. Umbral de sensibilidad del nervio radial izquierdo y su relación con edad. 


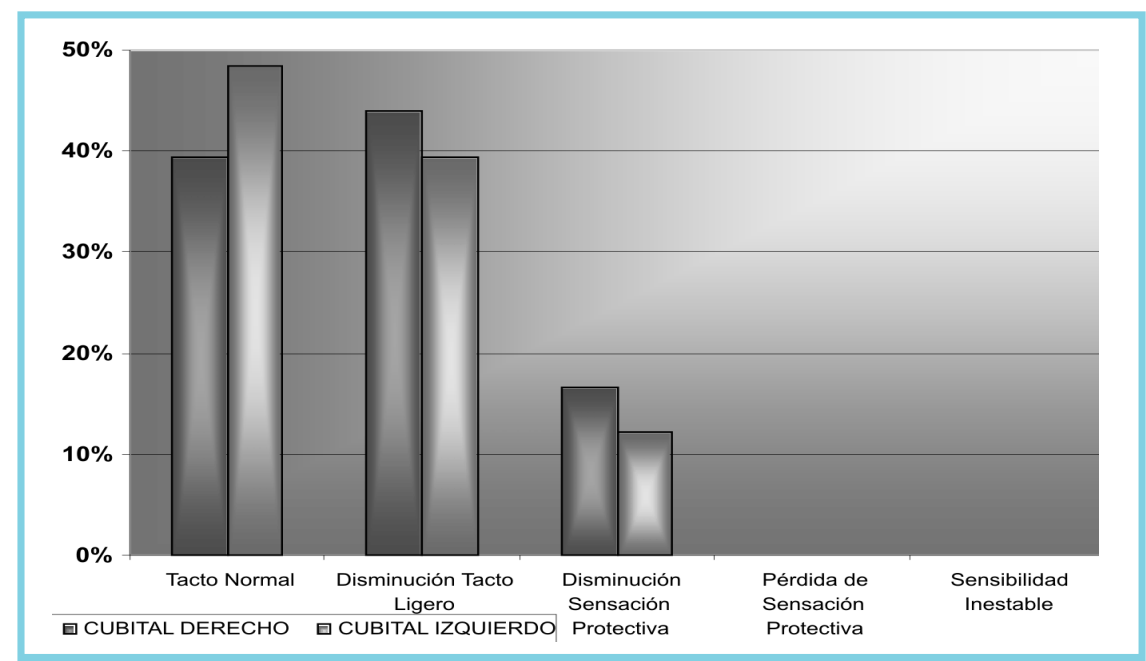

Gráfica 16. Umbral de sensibilidad de los nervios cubital derecho e izquierdo.

Todos los pacientes evaluados tenían lateralidad diestra y en el análisis comparativo de los nervios cubital derecho e izquierdo se encontró que el mayor porcentaje con $39,4 \%$ y $48,5 \%$ respectivamente, fue el tacto normal, seguido en ambos nervios por la disminución del tacto ligero con 43,9\% y 39,4\%. La disminución de la sensación protectiva también tuvo resultados similares con $16,7 \%$ y $12,1 \%$.
En igual porcentaje de 39\% los nervios mediano derecho e izquierdo tuvieron su mayor índice en el tacto normal seguido por la disminución del tacto ligero con $30 \%$ el mediano derecho y con $42 \%$ el mediano izquierdo. La sensación protectiva fue de $26 \%$ en mediano derecho y de $17 \%$ en mediano izquierdo. La pérdida de la sensación protectiva y la sensibilidad inestable tuvieron porcentajes de $3,0 \%$ y $2 \%$ respectivamente.

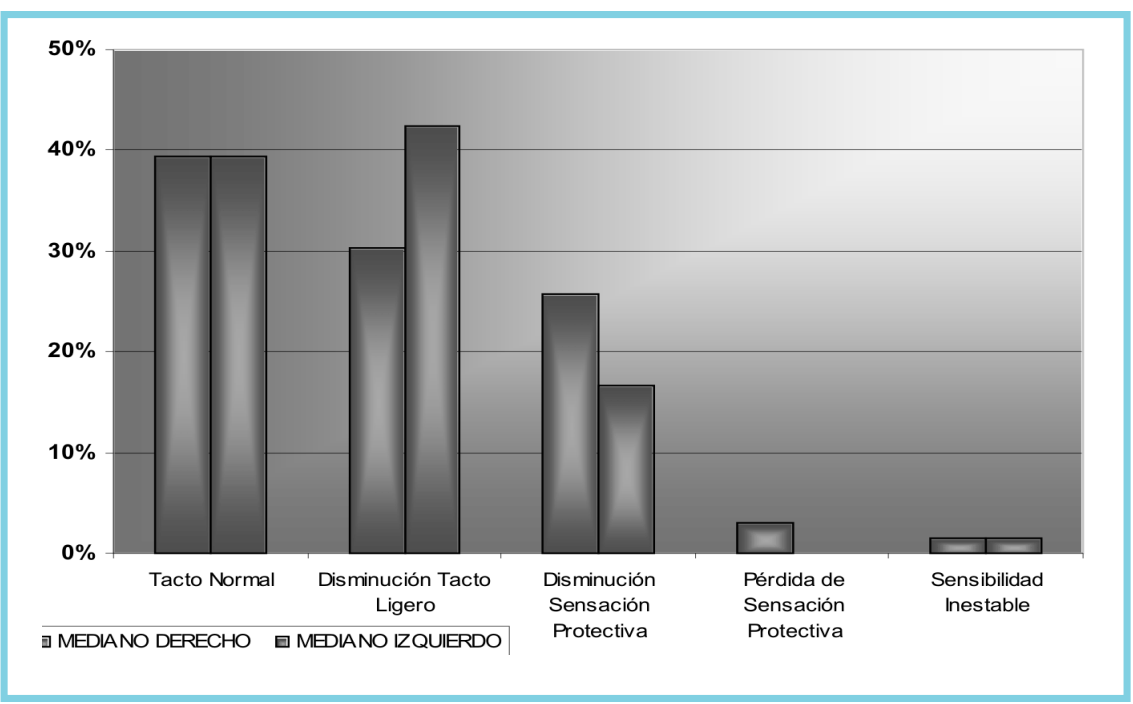

Gráfica 17. Umbral de sensibilidad de los nervios mediano derecho e izquierdo. 


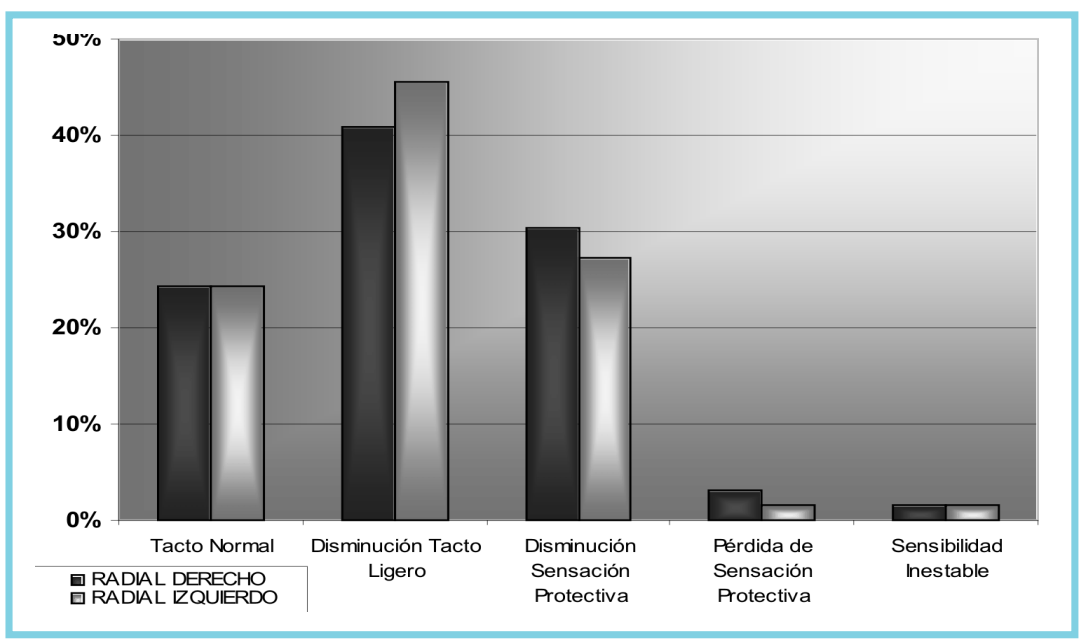

Gráfica 18. Umbral de sensibilidad de los nervios radial derecho e izquierdo.

$\mathrm{Al}$ evaluar los nervios radiales derecho e izquierdo se pudo establecer que en ambos hubo porcentajes similares de $41 \%$ y $45 \%$, respectivamente en la discriminación del tacto ligero, seguido por la disminución de la sensación protectiva con $30 \%$ y $27 \%$, también con porcentaje similar. Igual porcentaje de $24 \%$ se dio en el tacto normal. La pérdida de la sensación protectiva y la sensibilidad inestable tuvieron porcentajes de 3,0\% y $2 \%$.

\section{Discusión}

En la diabetes la neuropatía periférica es una de las complicaciones más frecuentes por compromiso vascular arterial, que no permite oxigenación de las diferentes estructuras con el consecuente deterioro en las fibras nerviosas tanto motoras como sensitivas, con consecuencias como la debilidad muscular y disminución o pérdida de la sensibilidad $^{19}$.

Con el fin de hacer aportes a la temática, esta investigación buscó comprobar el umbral de sensibilidad de los nervios de la mano, y para ello realizó una evaluación con monofilamentos, de las personas con diabetes tipo 2 que asistían a la Clí-

19 Waylet-Rendall, Janeth. Sensibility evaluation and rehabilitation. Orthopedics. Clinics of North America. January 1988, 19 (1). nica de Diabetes del HOSMIC, en 2007, que permitió establecer resultados específicos en cuanto a género, edad y dominancia y su relación con los nervios periféricos de la mano.

En cuanto a género, se encontró que de la muestra evaluada, el 42,4\% (29) pertenece al género masculino y el $57,6 \%$ (39) al de género femenino, lo cual es consistente con diferentes hallazgos bibliográfi$\cos ^{20},{ }^{21}$ que muestran la prevalencia de género femenino en el desarrollo de la diabetes tipo 2.

Respecto de la edad, en este estudio se pudo establecer que en el rango de edad de 70 a 74 años se presentan más alteraciones de umbral de sensibilidad de los tres nervios evaluados. Este resultado hace cuestionar si efectivamente, como consecuencia de la diabetes, los usuarios tienen neuropatías que llevan a alteraciones en el umbral de sensibilidad en manos, o si por el contrario, en este compromiso influye el proceso normal de envejecimiento.

20 Mata-Cases, Manuel y cols. Incidencia de diabetes tipo 2 y análisis del proceso diagnóstico en un centro de atención primaria durante la década de los noventa. Gac Sanit. Barcelona. 2006, 20 (2).

21 Lombo, Bernardo, Satizábal, Claudia, Villalobos, César. Prevalence of the metabolic syndrome in diabetic patients. Acta Med Colombiana. Ene-Mar. 2007, 32 (1): pp. 9-15. 
Todos los pacientes evaluados tuvieron lateralidad diestra y al comparar los resultados entre manos izquierdas y derechas no hubo diferencias importantes en el umbral de sensibilidad de los pacientes evaluados.

Los tres nervios, mostraron alto porcentaje en la disminución del tacto ligero. El nervio cubital fue el que tuvo el más alto porcentaje de normalidad en la prueba y en la disminución de la sensación protectiva el nervio radial fue el más comprometido.

Aunque hubo un alto porcentaje de normalidad en el umbral de sensibilidad de los nervios mediano y cubital, es necesario resaltar que en porcentajes superiores al $56 \%$ se encontró disminución de este umbral, lo cual debe tenerse en cuenta para prevenir complicaciones como lesiones del tejido blando de la mano y en etapas tardías, amputaciones.

\section{Conclusiones}

En los usuarios de la clínica de Diabetes del Hospital Militar Central (HOSMIC), en el segundo semestre de 2007, se encontró que en el umbral de sensibilidad de los nervios periféricos de la mano el mayor porcentaje corresponde a la disminución del tacto ligero con el 40,3\%.

La evaluación del umbral de sensibilidad del nervio cubital determinó que el mayor porcentaje fue el de tacto normal con el $44 \%$.

La evaluación del umbral de sensibilidad del nervio mediano mostró que el mayor porcentaje de los usuarios evaluados tenía tacto normal con $39 \%$, seguido de la disminución del tacto ligero, con $36 \%$.

La evaluación del umbral de sensibilidad en el nervio radial determinó mayor compromiso en la disminución del tacto ligero con un $43 \%$, seguido de la disminución de la sensación protectiva con un $29 \%$.
La edad de mayor compromiso en el umbral de sensibilidad se dio en usuarios mayores de 70 años, con el $22 \%$.

La evaluación del umbral de sensibilidad mostró que las mujeres presentan mayor porcentaje de tacto normal con $32 \%$ y los hombres tuvieron su mayor porcentaje en la disminución del tacto ligero, con $20 \%$.

La lateralidad diestra fue de $100 \%$ en los pacientes evaluados y comparativamente con la izquierda no se observó diferencia importante entre la mano derecha e izquierda de los umbrales de sensibilidad en los usuarios con diabetes tipo 2 del HOSMIC.

Recibido: agosto 2008

Aceptado: octubre 2008

\section{Bibliografía}

Brown J and Asbury AK. Diabetic neuropathy. Ann Neurol, 1984; 2-12.

Dimitrakoudis, Bril V. Comparation of sensory testing on different toe surfaces: Implications for neuropathy screening. Neurology, 2002.

Greene DA, Stevens MJ Feldman. Diabetic neuropathy. Scope of a syndrome. American Journal of Medicine. 1999; 3.

Hunter J. The hand: treatment and rehabilitation, Philadelphia. Ed. Mosby. 4ª ed. 1996.

Kamei $\mathrm{N}$ et al. Effectiveness of Semmes-Weinstein monofilament examination for diabetic peripheral neuropathy screening. Journal of Diabetes and its Complications. JanuaryFebruary 2005; 19.

Lee, S et al. Clinical Usefulness of Two-site Semmes-Weinstein Monofilament Test for Detecting Diabetic Peripheral Neuropathy. Korea. Journal Korean Medical Science. 2003; 18.

Lombo B, Satizábal C, Villalobos C. Prevalence of the metabolic syndrome in diabetic patients. 
Acta Med Colomb. Ene-mar. 2007; 32 (1): 9-15.

Mata-Cases $\mathrm{M}$ et al. Incidencia de diabetes tipo 2 y análisis del proceso diagnóstico en un centro de atención primaria durante la década de los noventa. Barcelona. Gaceta Sanitaria, 2006; 20 (2).

Mayfield JA. The use of the Semmes- Weinstein and other threshold tests for preventing foot ulceration and amputation in persons with diabetes. J Fam Pract. 2000; 49.

Meijer, Jan Willem et al. Clinical diagnosis of diabetic polyneuropathy with the diabetic neuropathy symptom and diabetic neuropathy examination scores. Diabetes Care. March 2003; 26 (3).

Organización Panamericana de la Salud. Iniciativa de diabetes para las Américas (DIA): Plan de acción para América Latina y el Caribe, 2001-2006. OPS y OMS 2001.

Organización Mundial de la Salud. La diabetes en las Américas. Boletín Epidemiológico, OMS. Junio de 2001; 22 (2).

Perkins BA. Simple screening tests for peripheral neuropathy in the diabetes clinic Diabetes Care. Feb. 2001; 2 (24): 250-256.

Programme: Diabetes Action Now. The role of World Health Organization in Geneva and how partners may support its work. Coordination of the joint WHO-IDF.

Secretaría de Salud Distrital. Manual de Norma Guía para el programa de prevención y control de la Diabetes Mellitus para Bogotá.
Secretaría Distrital de Salud de Bogotá, Dirección de Salud Pública, septiembre 2004.

Stanley B. Concepts in hand rehabilitation. Philadelphia. Ed. F.A. Davis Company. 2000.

Sullivan S. Assessment and treatment Physical Rehabilitation. Philadelphia. Davis Company. $4^{\mathrm{a}}$ ed. 2000.

Sumpio BE. Foot ulcers. The new England Journal of Medicine. 2000.

Travis, Luther B. Diabetes mellitus in children and adolescents. Philadelphia. Ed. Saunders. W.B. Company.1998.

Tubiana R. Examination of the hand and wrist. Primera edición. Mosby. 1998; 328.

Unwin N. Reducing the impact of diabetes on the World's poor. The role of World Health Organization in Geneva and how partners may support its work. Coordination of the joint WHO-IDF programme: Diabetes Action now. 2003.

Valk GD et al. Methods for assessing diabetic polyneuropathy: validity and reproducibility of the measurement of sensoy symptom severitu and nerve function tests. Institute for Research in Extramural Medicine, Vrije Universiteit, Amsterdam, 1999.

Waylet-Rendall J. Sensibility evaluation and rehabilitation. Orthopedics Clinics of North America. January 1988; 19 (1).

http://www.cbi pace.com/ monofilaments.com Monofilaments for evaluating sensory neuropathy. July 28, 2004. 